\title{
summary
}

\section{Little high-quality research into public water fluoridation}

\author{
McDonagh M, Whiting P, Bradley M, Cooper J, Sutton A, Chestnutt I, et al. A Systematic Review of Public Water \\ Fluoridation. York: Publications Office, NHS Centre for Reviews and Dissemination (CRD), University of York.
}

ISBN 190064016 3; 2000

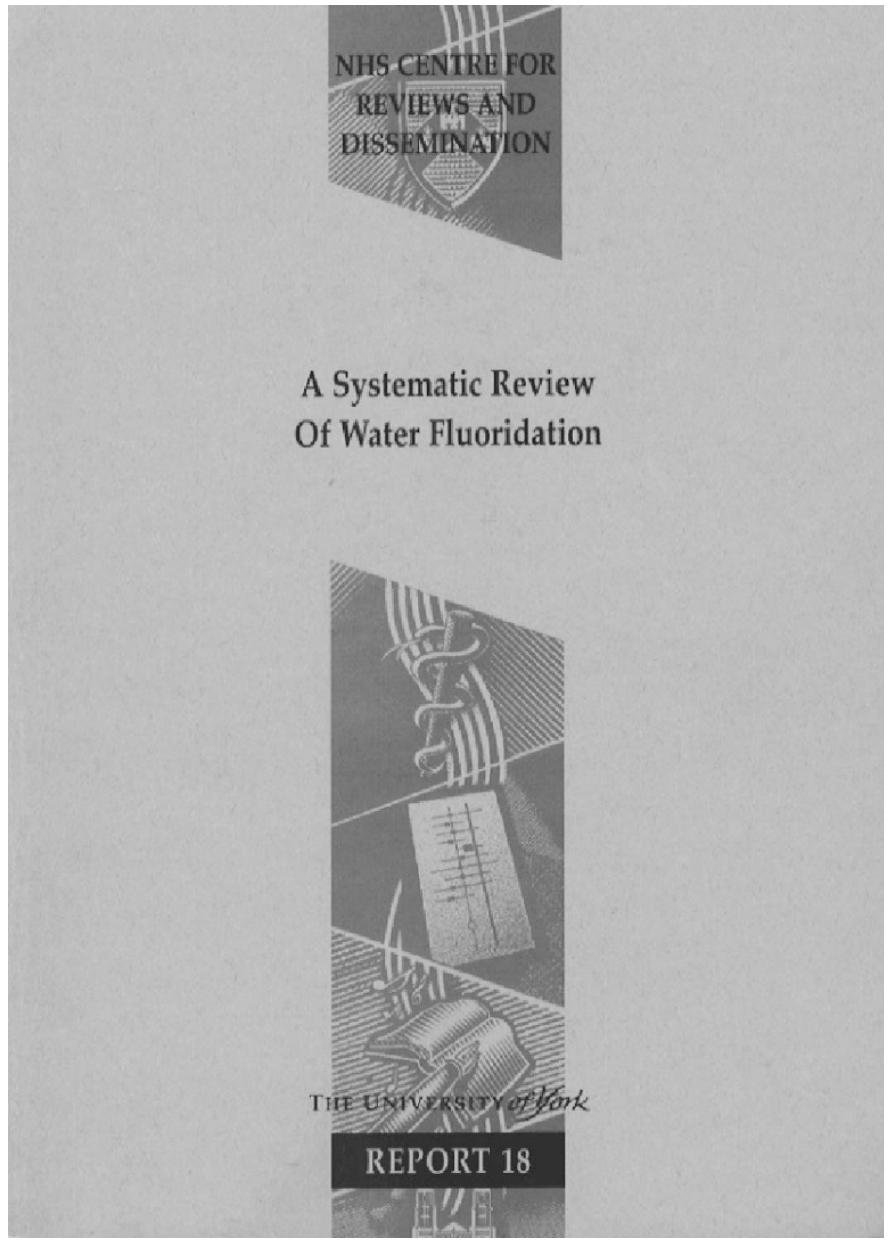

Aim To assess the evidence on the positive and negative effects of population-wide drinking-water fluoridation strategies to prevent caries. To achieve this aim, five objectives were identified:

Objective 1: What are the effects of fluoridation of drinking water supplies on the incidence of caries?

Objective 2: If water fluoridation is shown to have beneficial effects, what is the effect over and above that offered by the use of alternative interventions and strategies?

Objective 3: Does water fluoridation result in a reduction of caries across social groups and between geographical locations, bringing equity?

Objective 4: Does water fluoridation have negative effects?

Objective 5: Are there differences in the effects of natural and artificial water fluoridation?
Data sources A search of 25 electronic databases with no language restrictions and of the World-Wide-Web was undertaken. Databases used were Medline, Embase, National Technical Information Service, Biosis Current Contents Search (Science Citation Index and Social Science Citation Index), Health Service Technology, Administration and Research, HSRProj, TOXLINE Chemical Abstracts, OldMedline, CAB Health, Food Science and Technology Abstracts, JICST E Plus (Japanese Science and Technology), Pascal, Engineering Index Compendex, Enviroline, Public Affairs Information Services, System for Information on Grey Literature in Europe, Conference Papers Index, Water Resources Abstracts, Agricola (Agricultural Online Access), Waternet, Allied and Complementary Medicine Database, Psyclit and LILACS (Latin American and Caribbean Health Sciences Literature). Relevant journals and indices were searched by hand and attempts were made to contact authors for further information.

Study selection Quality inclusion criteria were based on a predefined hierarchy of evidence (A, B and C; see Table). Studies of efficacy were included if they were of evidence level A or B. In order to allow the broadest search for evidence on potential adverse effects, studies of all levels of evidence were included. Objective-specific inclusion criteria, based on selection of participants, intervention,

Table Heirarchy of evidence for study inclusion.

Level A Highest quality of evidence, minimal risk of bias

Prospective studies begun $<1$ year after initiation/ discontinuation of water fluoridation with follow-up of $\geqslant 2$ years for positive effects and $\geqslant 5$ years for negative effects Studies randomised, or addressing $\geqslant 3$ possible confounding factors and adjusting for these in analysis as appropriate Studies where fluoridation status of participants is unknown to those assessing outcomes

Level B Evidence of moderate quality, moderate risk of bias Studies started $<3$ years after initiation/discontinuation of water fluoridation with prospective follow-up for outcomes Studies that measured and adjusted for $<3$ but $\geqslant 1$ confounding factor

Studies in which fluoridation status of participants was known to those assessing primary outcomes, but other provisions were made to prevent measurement bias

Level C Lowest quality of evidence, high risk of bias

Studies of other designs, eg, cross-sectional, prospective/ retrospective, using concurrent or historical controls, or meeting other inclusion criteria

Studies that failed to adjust for confounding factors Studies that did not prevent measurement bias

Studies meeting two of the three criteria for a given evidence level were assigned the next level down, eg, if a study met the criteria for prospective design and blinding for level $A$, but was neither randomised nor controlled for $\geqslant 3$ potential confounding factors, it was assigned to level B. 
outcomes assessed and study design appropriate for a given objective were then applied. Study validity was formally assessed using a published checklist modified for this review. ${ }^{1}$ Inclusion criteria were assessed independently by at least two reviewers. Extraction of data and validity assessment of included studies was independently performed by two reviewers, and checked by a third reviewer. Disagreements were resolved through consensus.

Data extraction and synthesis Where the data were available in a suitable format, measures of effect and 95\% confidence intervals were plotted. Heterogeneity was investigated by visual examination and statistically using the Q-statistic. Where no evidence of heterogeneity was found a meta-analysis was conducted to produce a pooled estimate of the measure of effect. Statistically significant heterogeneity was investigated using meta-regression. Multiple regression analysis was used to explore the relationship between fluoridation and fluorosis.

Results Two hundred and fourteen studies met full inclusion criteria for one or more of the objectives. No randomised controlled trials of the effects of water fluoridation were found. The study designs included 45 'before and after' studies, 102 cross-sectional studies, 47 ecological studies, 13 cohort (prospective or retrospective) studies and seven case-control studies. Several studies were reported in multiple papers over a number of years.

Conclusions This review presents a summary of the best available and most reliable evidence on the safety and efficacy of water fluoridation. Given the level of interest surrounding the issue of public water fluoridation, it is surprising to find that little highquality research has been undertaken. This review should provide both researchers and those commissioning research with an overview of the methodological limitations of previous research conducted in this area. The evidence of a benefit of a reduction in caries should be considered together with the increased prevalence of dental fluorosis. The research evidence is of insufficient quality to allow confident statements about other potential harms or whether there is an impact on social inequalities. This evidence on benefits and harms needs to be considered along with the ethical, environmental and ecological costs and legal issues that surround any decisions about water fluoridation. All of these issues fall outside the scope of this review. Any future research into the safety and efficacy of water fluoridation should be carried out with appropriate methods to improve the quality of the existing evidence base.

Evidence-Based Dentistry (2002) 3, 37-38. doi:10.1038/sj. ebd.6400104

Address for reprints: Publications Office, NHS Centre for Reviews and Dissemination, University of York, York YO10 5DD, UK.

Tel.: +44 (0)1904 433648; Fax: +44 (0)1904 433661;

E-mail: crdpub@york.ac.uk; http://www.york.ac.uk/inst/crd/fluorid.htm.

1. NHS Centre for Reviews and Dissemination. Undertaking Systematic Reviews of Research on Effectiveness: CRD's Guidance for Carrying Out or Commissioning Reviews (2nd Edition). NHS CRD, University of York, York, 2001 